\title{
Interspecific hybridization in natural sturgeon populations of the Eastern Black Sea: the consequence of drastic population decline?
}

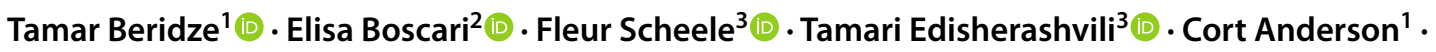 \\ Leonardo Congiu ${ }^{2}$ (B)
}

Received: 10 February 2021 / Accepted: 14 October 2021 / Published online: 30 October 2021

(c) The Author(s) 2021

\begin{abstract}
The eastern part of the Black Sea and its tributaries are suitable habitats for several sturgeon species, among which Acipenser gueldenstaedtii, A. stellatus, A. nudiventris, A. persicus, A. sturio, and H. huso are well documented. However, different threats have led these species to a dramatic decline, all of them are currently listed as Critically Endangered, and some Locally Extinct, in that area. We tested 94 wild sturgeon samples from the Black Sea and Rioni River by analyzing the mitochondrial Control Region and nuclear markers for hybrid identification. The data analyses (1) assessed mitochondrial diversity among samples, (2) identified their species, as well as (3) indicated instances of hybridization. The data collected, besides confirming a sharp decrease of catches of Beluga and Stellate sturgeon in recent years, also revealed four juvenile hybrids between Russian and Stellate sturgeon, providing the first evidence of natural interspecific hybridization in the Rioni. The present communication raises concerns about the status of sturgeon species in this area and underlines the urgent need for conservation programs to restore self-sustaining populations.
\end{abstract}

Keywords Sturgeons $\cdot$ Interspecific hybrids $\cdot$ Acipenser gueldenstaedtii $\cdot$ Acipenser stellatus $\cdot$ Rioni $\cdot$ Black Sea

\section{Introduction}

Sturgeons are among the most endangered species groups in the world according to the International Union for Conservation of Nature (IUCN 2010), with some of the most

Tamar Beridze, Elisa Boscari have contributed equally to this work.

Fleur Scheele

fleur.scheele@fauna-flora.org

Elisa Boscari

elisa.boscari@unipd.it

Tamari Edisherashvili

tamar.edisherashvili@fauna-flora.org

Leonardo Congiu

leonardo.congiu@unipd.it

1 School of Natural Sciences and Medicine, Ilia State University, Tbilisi, Georgia

2 Department of Biology, University of Padua, Padua, Italy

3 Fauna \& Flora International, Tbilisi, Georgia imperiled species distributed in the Palearctic region. The Eastern part of the Black Sea and a major tributary in the Caucasus, the Rioni River, are known to have hosted in historical times at least five sturgeon species. The Russian sturgeon (Acipenser gueldenstaedtii), the Stellate sturgeon ( $A$. stellatus), the Beluga sturgeon (Huso huso), the European sturgeon (A. sturio), and the Ship sturgeon (A. nudiventris) (Variadilis et al. 1998; Guchmanidze 2009). All of these are listed by IUCN as Critically Endangered, with European and Ship sturgeons also believed to be Locally Extinct, with wild populations considered to be extirpated from the Black Sea basin (Gessner et al. 2010; Mugue et al. 2016).

The population decline is mainly caused by habitat degradation, including river damming and consequent high sediment flushing, overfishing, and pollution. Accurate historical or present assessments of population sizes are not available, but there are indications that sturgeon populations in the region have been in steep decline since the early 20th century (Beridze et al. 2021). Historically, the Rioni River in Georgia is known as one of the main sites for sturgeon spawning in this area (IUCN, 2010, www.iucnredlist.org) and it currently is the only remaining functional sturgeon 
spawning river of the Eastern Black Sea. This was confirmed by monitoring research on sturgeon recruitment in the Rioni conducted by Fauna \& Flora International (FFI) between 2018 and 2020. Intensive field surveys, annually held from March until October, led to the discovery of multiple juvenile sturgeon specimens of various species. In addition, data collection with associated anglers on the Rioni led to the discovery of eight specimens of A. nudiventris in 2020. Their possible origin from an ongoing captive breeding program in Krasnodar (River Kuban) has been excluded, supporting the hypothesis of a relict reproductively active population of A. nudiventris in the Rioni River (Beridze et al. 2021).

This study reports the results of the genetic characterization of sturgeons collected within the first three years of these monitoring activities. Monitoring is ongoing, with the aim of evaluating the state of sturgeon populations of this area and verifying abundance of the different species, the purity of the animals and whether the sampled individuals come from restocking activities or if they result from natural reproduction.

The collected data has unexpectedly indicated the occurrence of interspecific hybrids between two species of sturgeon historically known to reproduce in the Rioni River. The occurrence of interspecific hybridization events is discussed in light of implications for the conservation of natural sturgeon populations.

\section{Materials and methods}

Between 2018 and 2020, a sturgeon sampling campaign was carried out in the Georgian part of the Black Sea (BS) and Rioni River (RR = Rioni River; RM = Rioni mouth) (Fig. 1A) by FFI. A total of 94 tissue samples were collected (Table 1); captured animals, mostly juveniles, were immediately released after sampling.
Fig. 1 Study area, sampling locations, and haplotype relationships. A Geographic locations of sampled individuals (green $=A$. stellatus, yellow $=$ A. gueldenstaedtii, and blue = $H$. huso). The inset map shows a continental view where the sampling area in the southeastern Black Sea is framed. Satellite image obtained from Google Earth Pro V. 7.3.3.7786 (Google LLC, California, USA). B-D Haplotype networks, obtained with PopART 1.7 software, showing relationships among haplotypes in the three species detected. The size of pie charts is proportional to the corresponding haplotype frequency while colours indicate the sampling origin (Yellow = BS - Black Sea; Blue $=$ RR Rioni River, Red $=\mathrm{RM}-$ Rioni Mouth). Capital letters B, C, and $\mathrm{D}$ show haplotype relationships of A. gueldenstaedtii (GUE), A. stellatus (STE), and H. huso (HUS), respectively. E) Schematic NJ tree representing haplotypic distances between species

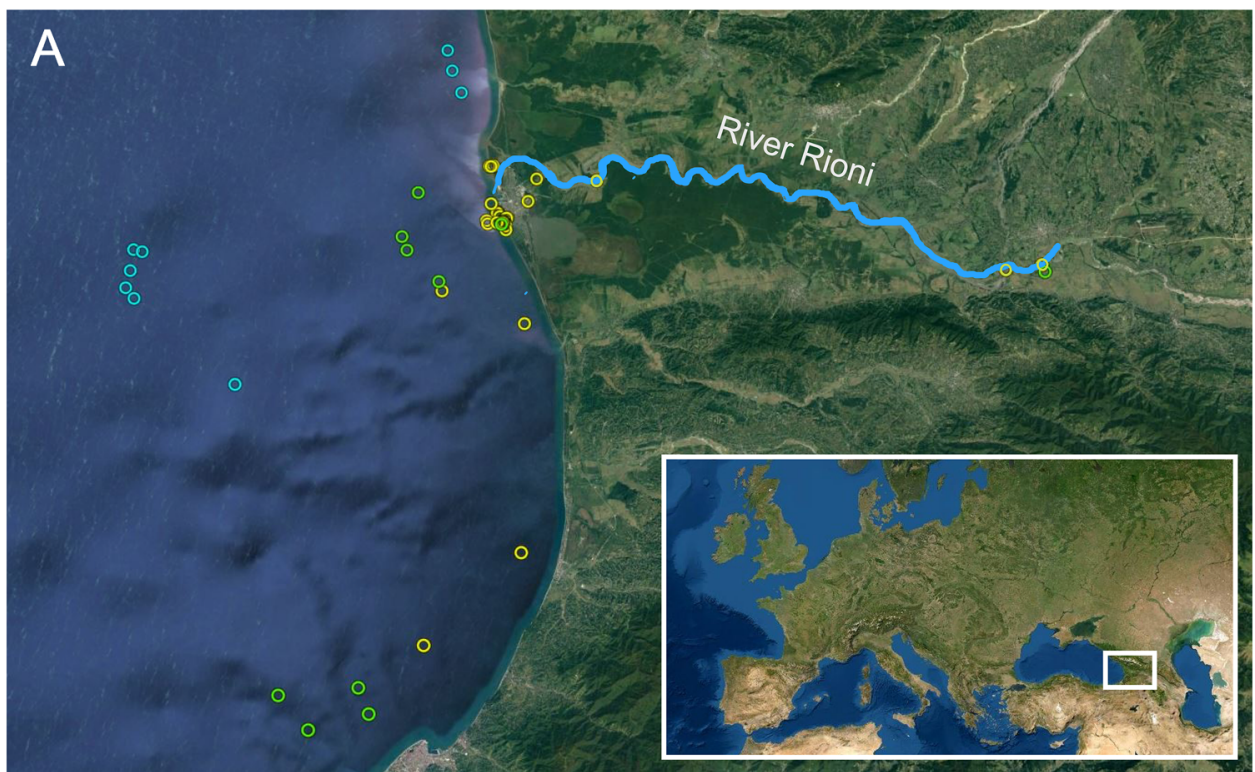

B

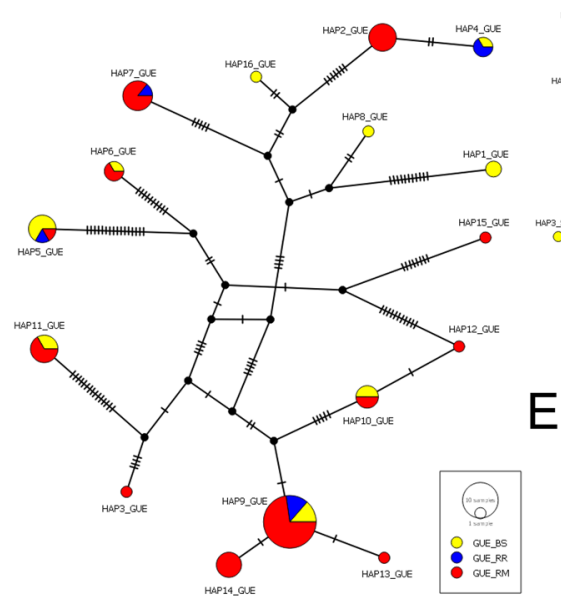

D

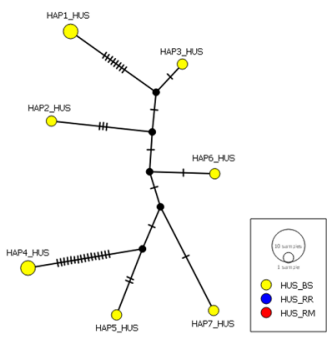

E

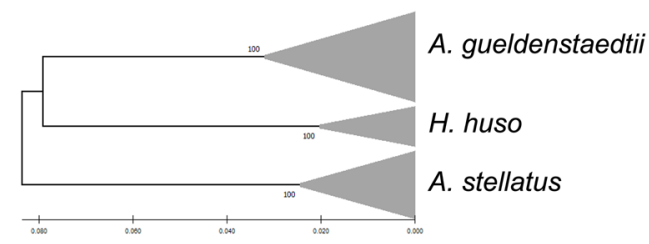


Table 1 Summary of the results from mitochondrial control region and diagnostic nuclear markers which specifically identify A. stellatus (ste), A. gueldenstaedtii (gue) and $H$. huso (hus)

\begin{tabular}{|c|c|c|c|c|c|c|c|}
\hline \multirow[t]{2}{*}{ Year } & \multirow[t]{2}{*}{ SampleID } & \multirow{2}{*}{$\begin{array}{c}\text { mtDNA } \\
\text { (present study) }\end{array}$} & \multicolumn{2}{|c|}{$\begin{array}{l}\text { Match with previously known } \\
\text { haplotypes }\end{array}$} & \multicolumn{3}{|c|}{$\begin{array}{l}\text { Nuclear } \\
\text { markers }\end{array}$} \\
\hline & & & Hap/Acc.n. & Occurence & ste & gue & hus \\
\hline \multirow[t]{5}{*}{2018} & Ac91_BS & ste_Hap1 & STE-HAP21 & AS & & & \\
\hline & Ac86_RR & ste_Hap2 & STE-HAP108 & CS & & & \\
\hline & Ac89_BS & ste_Hap3 & I & I & & & \\
\hline & Ac92_BS, Ac105_BS & ste_Hap4 & I & I & & & \\
\hline & Ac94_RM, Ac114_RM & ste_Hap5 & STE-HAP83 & AS & & & \\
\hline \multirow[t]{7}{*}{2019} & Ac137_RM & ste_Hap5 & STE-HAP83 & AS & & & \\
\hline & Ac101_BS & ste_Hap6 & AF168525* & 1 & & & \\
\hline & Ac115_RM & ste_Hap7 & STE-HAP62 & AS & & & \\
\hline & Ac123_BS & ste_Hap8 & AF168528* & I & & & \\
\hline & Ac139_BS & ste_Hap9 & AF168535* & 1 & & & \\
\hline & Ac157_BS & ste_Hap10 & STE-HAP15 & CS/UR & & & \\
\hline & Ac156_BS & ste_Hap11 & STE_HAP64 & DR/UR & & & \\
\hline 2020 & Ac193_BS & ste_Hap12 & STE_HAP9 & AS & & & \\
\hline \multirow[t]{5}{*}{2018} & Ac70_BS, Ac76"\#_BS & gue_Hap1 & 1 & 1 & & & \\
\hline & Ac93_RM, Ac95_RM & gue_Hap2 & GUE_HAp137 & BS & & & \\
\hline & Ac96_RM & gue_Hap3 & I & l & & & \\
\hline & Ac112_RR & gue_Hap4 & l & l & & & \\
\hline & Ac113_RR & gue_Hap5 & $\begin{array}{l}\text { HQ7304710 } \\
\text { GUE_HAP195 } \\
\end{array}$ & $\mathrm{BS} / \mathrm{CS}$ & & & \\
\hline \multirow[t]{10}{*}{2019} & Ac125_BS & gue_Hap4 & 1 & / & & & \\
\hline & Ac120_BS & gue_Hap5 & $\begin{array}{l}\text { HQ7304710 } \\
\text { GUE_HAP195 }\end{array}$ & $\mathrm{BS} / \mathrm{CS}$ & & & \\
\hline & Ac116_RM, Ac136_RM & gue_Hap6 & GUE_HAP101 & AS/BS & & & \\
\hline & $\begin{array}{l}\text { Ac118_RR, Ac144_RM, } \\
\text { Ac145_RM }\end{array}$ & gue_Hap7 & 1 & I & & & \\
\hline & Ac119_BS & gue_Hap8 & GUE_HAP1 & $\mathrm{BS} / \mathrm{CS}$ & & & \\
\hline & $\begin{array}{l}\text { Ac121_BS, Ac135_RR, } \\
\text { Ac143_RM, Ac146_RM, } \\
\text { Ac147_RM, Ac148_RM }\end{array}$ & gue_Hap9 & $\begin{array}{l}\mathrm{AF} 238725^{\circ} \\
\text { GUE_HAP11 }\end{array}$ & AS/BS & & & \\
\hline & Ac127_BS & gue_Hap10 & GUE_HAP140 & $\mathrm{DR}$ & & & \\
\hline & Ac128_BS, Ac140_RM & gue_Hap10 & GUE_HAP140 & DR & & & \\
\hline & Ac133_BS & gue_Hap11 & GUE_HAP135 & BS & & & \\
\hline & Ac150_RM & gue_Hap11 & GUE_HAP135 & BS & & & \\
\hline \multirow[t]{16}{*}{2020} & $\begin{array}{l}\text { Ac198_RM, Ac200_RM, } \\
\text { Ac203_RM, Ac205_RM }\end{array}$ & gue_Hap2 & GUE_HAp137 & BS & & & \\
\hline & Ac196_RM & gue_Hap4 & I & I & & & \\
\hline & $\begin{array}{l}\text { Ac189_BS, Ac194_BS, Ac210_BS, } \\
\text { Ac201_RM }\end{array}$ & gue_Hap5 & $\begin{array}{l}\text { HQ7304710 } \\
\text { GUE_HAP195 }\end{array}$ & $\mathrm{BS} / \mathrm{CS}$ & & & \\
\hline & Ac199_BS & gue_Hap6 & GUE_HAP101 & AS/BS & & & \\
\hline & $\begin{array}{l}\text { Ac171_RM, Ac182_RM, } \\
\text { Ac185_RM, Ac188_RM }\end{array}$ & gue_Hap7 & I & I & & & \\
\hline & $\begin{array}{l}\text { Ac204_BS, Ac209_BS, Ac165_RR, } \\
\text { Ac161_RM, Ac163_RM, } \\
\text { Ac168_RM, Ac172_RM, } \\
\text { Ac174_RM, Ac177_RM, } \\
\text { Ac179_RM, Ac181_RM, }\end{array}$ & gue_Hap9 & $\begin{array}{l}\text { AF238725 } \\
\text { GUE_HAP11 }\end{array}$ & AS/BS & & & \\
\hline & $\begin{array}{l}\text { Ac183_RM, Ac187_RM, } \\
\text { Ac202_RM, Ac208_RM }\end{array}$ & & & & & & \\
\hline & Ac160_RR & gue_Hap9 & $\begin{array}{l}\text { AF238725 } \\
\text { GUE_HAP11 }\end{array}$ & AS/BS & & & \\
\hline & Ac197_RM & gue_Hap10 & GUE_HAP140 & $\mathrm{DR}$ & & & \\
\hline & Ac175_BS & gue_Hap11 & GUE_HAP135 & BS & & & \\
\hline & $\begin{array}{l}\text { Ac162_RM, Ac164_RM, } \\
\text { Ac173_RM }\end{array}$ & gue_Hap11 & GUE_HAP135 & BS & & & \\
\hline & Ac167_RM & gue_Hap12 & GUE_HAP140 & DR/UR & & & \\
\hline & Ac169_RM & gue_Hap13 & 1 & I & & & \\
\hline & $\begin{array}{l}\text { Ac170_RM, Ac176_RM, } \\
\text { Ac180_RM, Ac186_RM, } \\
\text { Ac192_RM }\end{array}$ & gue_Hap14 & GUE_HAP185 & CS & & & \\
\hline & Ac207_RM & gue_Hap15 & 1 & I & & & \\
\hline & Ac212_BS & gue_Hap16 & GUE_HAP141 & $\mathrm{DR} / \mathrm{CS}$ & & & \\
\hline \multirow[t]{3}{*}{2018} & Ac72_BS, Ac90_BS & hus_Hap1 & "HUS_HAP6O & $\overline{\mathrm{DR} / \mathrm{CS}}$ & & & \\
\hline & Ac73_BS & hus_Hap2 & HUS_HAP73 & DR & & & \\
\hline & Ac74_BS & hus_Hap3 & I & I & & & \\
\hline \multirow[t]{4}{*}{2019} & Ac98_BS, Ac99_BS & hus_Hap4 & l & I & & & \\
\hline & Ac100_BS & hus_Hap5 & HUS_HAP12 & $\mathrm{AS} / \mathrm{BS} / \mathrm{CS}$ & & & \\
\hline & Ac102_BS & hus_Hap6 & I & / & & & \\
\hline & Ac104_BS & hus_Hap7 & HUS_HAP75 & DR & & & \\
\hline
\end{tabular}


Table 1 (continued)

Samples are sorted by collecting year and by haplotype. Any correspondence with known haplotypes and their distribution is also reported. Codes in italics indicate haplotypes detected in the Russian sturgeon broodstocks. For each sample, the amplification of diagnostic nuclear markers is shown in grey-filled cells. Amplification of nuclear markers from four individuals failed, indicated in the table by the empty cells

$B S$ Black Sea, RR Rioni River, $R M$ Rioni mouth, AS Azov Sea, DR Danube River, CS Caspian Sea

*Doukakis et al 1999; ${ }^{\circ}$ Birstein et al. 2000; \#= A. gueldenstaedtii baerii-like haplotype

Genomic DNA was purified using the Qiagen DNeasy Blood \& Tissue Kit. All samples were genetically analyzed for species and hybrid identification by amplifying and sequencing the mitochondrial control region, and by checking the presence/absence of sturgeon diagnostic nuclear markers.

Primer pairs, PCR amplifications, and thermocycler conditions for the control region are as reported in the original reference by Congiu et al. (2011). PCR reactions were performed on Applied Biosystem GeneAmp®PCR System 9700 and MJ Research PTC-225 thermal cyclers. All PCR products were purified with ExoSAP- IT ${ }^{\circledR}$ according to the manufacturer's protocol, and directly sequenced on an ABI Prism 3730XL or an ABI 3100 automatic sequencer at Eurofins Genomics (Germany) or an ABI Prism 3730XL automatic sequencer at Macrogen Europe B.V. (Netherlands).

Mitochondrial sequences were aligned using ClustalW in MegaX (Kumar et al. 2018) and BLAST (Basic Local Alignment Search Tool, Altschul et al. 1990) searches were performed to determine the maternal species; most individuals were juveniles, making morphological identification more difficult. Mitochondrial genetic variation among collected samples was also evaluated. Haplotype diversity (h) and nucleotide diversity $(\pi)$ were estimated with ARLEQUIN ver.3.5 (Excoffier and Lischer 2010) for each group of species detected by the BLAST searches.

Haplotypes and their relationships (i.e., representation of gene genealogies based on a maximum parsimony approach) were organized in networks with the PopART 1.7 software (Leigh and Bryant 2015; http://popart.otago. ac.nz) based on TCS network inference methods (Clement et al. 2000). A schematic neighbor-joining tree based on p-distance was generated by MegaX. Haplotypes were also compared with available datasets, including information on haplotype diversity of wild and captive sturgeon populations collected over the past years (personal communication by N. Mugue).

Focusing on sturgeon species that more likely could hybridize in the Black Sea and Rioni River, available diagnostic nuclear markers for A. stellatus (Ste_RP1F and RP1_LocusA_R, Boscari et al. 2014), A. gueldenstaedtii (395_AB_for and 395_uni, Havelka et al. 2019), and $H$. huso (RP2S6_huso-F and RP2S6_groupA_R, Boscari et al. 2017) were also used to test samples for interspecific hybridization. Experimental protocols were as reported in the original references.

The presence/absence of diagnostic products (479 bp for A. stellatus, $395 \mathrm{bp}$ for A. gueldenstaedtii, and 194 for H. huso) was checked on $1.8 \%$ agarose gel stained with GelRed (BIOTIUM, GelRed ${ }^{\mathrm{TM}}$ Nucleic Acid Stain).

\section{Results and discussion}

BLAST performed with mitochondrial data revealed three species: A. gueldenstaedtii (Accession numbers: MZ665962MZ665977), A. stellatus (MZ665978-MZ665989), and $H$. huso (MZ665990-MZ665996) (Table 1), with 74\% of haplotypes indicating gueldenstaedtii species identification. Among samples collected from the Black Sea (BS), 10 animals presented A. stellatus haplotypes, 17 A. gueldenstae$d t i i$, and nine $H$. huso. In the Rioni River and its mouth (RR and RM) only A. stellatus (one RR samples and four RM samples) and A. gueldenstaedtii (six RR samples and 47-RM samples) haplotypes were found.

For each sample, Table 1 shows year of collection and the previous detection of each haplotype in wild populations or captive stocks; results of tests for interspecific hybridization performed with diagnostic nuclear markers are also indicated. Table 2 describes mitochondrial diversity for the three species. Figure 1B-E shows the relationships among haplotypes in the three species (12 haplotypes for A. stellatus, 16 for $A$. gueldenstaedtii, and seven for $H$. huso). For the three species, two, nine, and three haplotypes respectively were never observed before.

Four individuals with A. gueldenstaedtii haplotypes (one young of the year caught in 2018 and three 1 year olds caught in 2020), were positive for the diagnostic nuclear marker for A. stellatus, strongly pointing to their hybrid

Table 2 Summary data based on control region sequences

\begin{tabular}{lrrlll}
\hline \multicolumn{6}{l}{ Control Region summary basic statistics } \\
\hline Maternal species & \multicolumn{1}{c}{$\mathrm{N}$} & \multicolumn{1}{c}{$\mathrm{N}_{\mathrm{h}}$} & Ps & $\mathrm{h}$ & \multicolumn{1}{l}{$\pi$} \\
\hline A. stellatus & 15 & 12 & 52 & $0.962 \pm 0.040$ & $0.019 \pm 0.010$ \\
A. gueldenstaedtii & 70 & 16 & 87 & $0.867 \pm 0.029$ & $0.023 \pm 0.011$ \\
H. huso & 9 & 7 & 36 & $0.944 \pm 0.070$ & $0.017 \pm 0.009$ \\
\hline
\end{tabular}

$N$ number of individuals, $N h$ number of haplotypes, $P$ s Polymorphic sites, $h$ haplotype diversity, $\pi$ nucleotide diversity 
origin. It is worth noting that, for one of these animals, an informal identification as A. stellatus was provided before release by the FFI team members who collected it. The clear discordance between this a priori morphological classification and the haplotype sequence likewise indicates hybrid origin of that individual. Moreover, even though morphological indications for the other three detected hybrids were not provided, the A. stellatus diagnostic marker has never given false positive amplification in 11 other sturgeon species, including amongst 41 specimens of A. gueldenstaedtii from outside of the Black Sea (Boscari et al. 2014), strongly corroborating the reliability of this result. Given the underrepresentation of reference Russian sturgeons from the Black Sea, we cannot exclude that the allele frequency differs across locations such that $A$. gueldenstaedtii in the Black Sea might naturally carry the A. stellatus-diagnostic allele. However, commercial controls for species purity we routinely performed also included many A. gueldenstaedtii caviar samples from the Black Sea which have never shown the A. stellatus allele (data not shown).

The four haplotypes of the hybrid animals were not detected in the Russian sturgeon broodstocks used to generate juveniles for restocking in North Eastern Black Sea (Nikolai Mugue, pers. comm), suggesting that the hybrids found in this study were likely the offspring of wild breeders. Additionally, the four putative hybrids exhibited different haplotypes, indicating that each had a different A. gueldenstaedtii mother, and that hybridization involved at least four females. Sturgeons are known for their ability to hybridize in captivity and several species combinations have been generated in aquaculture for production purposes (Boscari et al. 2014). To our knowledge however, hybrids between $A$. gueldenstaedtii and A. stellatus are not used in aquaculture, and this species combination is not produced. Furthermore, no hatchery producing A. stellatus, either as pure species or as hybrids, is present in the area, excluding the possibility that the detected hybrids represent accidental escapees from aquaculture plants. This is not the first evidence of interspecific hybridization in nature between sturgeon species; for example, natural interspecific hybrids between A. ruthenus and $A$. baerii were found in the Danube River following careless release of the allochthonous A. baerii (Ludwig 2009). In this instance, it appears that hybridization occurs between indigenous species.

Although, a certain rate of hybridization may have always occurred, the present low density of populations (in particular of the Stellate sturgeon) might increase this phenomenon. Population decline may in fact promote interspecific hybridization due to the scarcity of conspecific mates. This phenomenon, known as Hubb's 'desperation' hypothesis (Hubbs 1955), adduces the urgent need for sturgeon conservation measures in the Eastern Black Sea and Rioni drainage. Additional concerns are raised by the possible impact that the presence of interspecific hybrids might have on the already seriously compromised natural populations (Havelka et al. 2011). In our specific case, the two parental species have respectively about 240 (Russian sturgeon) and 120 chromosomes (Stellate sturgeon) and the resulting hybrids, having an intermediate chromosomal set, are expected to be sterile (Birstein 2002; Linhartová et al. 2018). However, the sterile condition does not prevent the animals from taking part to spawning as adults, competing with breeders of pure species with adverse effects on their reproductive success (Arcella et al. 2014; Fjelldal et al. 2014). Furthermore, a relict population of Ship sturgeon (A. nudiventris), whose chromosomal set is compatible with the Stellate sturgeon (A. stellatus), also inhabits the Rioni River (Beridze et al. 2021). An interspecific admixture between these species would result in fertile hybrid which might backcross with the parental species, potentially compromising their genetic integrity. This would be particularly harmful for the Ship sturgeon, on the verge of being classified as extinct in the wild, and for which a last spawning site of the entire distribution area was recently recorded in the River Rioni (Beridze et al. 2021).

Acknowledgements We would like to acknowledge the contributions of Fauna \& Flora International's sturgeon conservation team and their associated fishers who collected the samples used in this project. We thank Dr. Nikolai Mugue, Dr. Natalia Nebesikhina and Viktoria Scherbakova for comparing detected haplotypes with Russian database of captive broodstocks.

Authors' contributions TB and EB equally contributed to this study. FS, CA substantially contributed to the design of the work. TB and EB performed all the experiments and data analyses. TB, EB and LC contribute to results interpretation and wrote the manuscript. All the authors critically revised the manuscript and accepted the final version for publication.

Funding This work was financially supported by U.S. Fish and Wildlife Service with Federal Grant number F19AP00450 to Fauna \& Flora International, by the UK Government through the Illegal Wildlife Trade Challenge Fund, grant number IWT082 to Fauna \& Flora International, and by Shota Rustaveli National Science Foundation (SRNSF) [PHDF_19_532, Molecular taxonomy, phylogeny, and population genetic structure of protected sturgeon species in Georgia].

Availability of data and material Accession numbers available upon acceptance.

Code availability Not applicable.

\section{Declarations}

Conflicts of interest/Competing interests No conflict of interest.

Ethical approval Not applicable.

Consent to participate All authors have given consent to participate.

Consent for publication All authors have given consent to publication. 
Open Access This article is licensed under a Creative Commons Attribution 4.0 International License, which permits use, sharing, adaptation, distribution and reproduction in any medium or format, as long as you give appropriate credit to the original author(s) and the source, provide a link to the Creative Commons licence, and indicate if changes were made. The images or other third party material in this article are included in the article's Creative Commons licence, unless indicated otherwise in a credit line to the material. If material is not included in the article's Creative Commons licence and your intended use is not permitted by statutory regulation or exceeds the permitted use, you will need to obtain permission directly from the copyright holder. To view a copy of this licence, visit http://creativecommons.org/licenses/by/4.0/.

\section{References}

Altschul SF, Gish W, Miller W, Myers EW, Lipman DJ (1990) Basic local alignment search tool. J Mol Biol 215:403-410. https://doi. org/10.1016/S0022-2836(05)80360-2

Arcella TE, Perry WL, Lodge DM, Feder JL (2014) The role of hybridization in a species invasion and extirpation of resident Fauna: hybrid vigor and breakdown in the Rusty Crayfish, Orconectes Rusticus. J Crustac Biol 34:157-164. https://doi.org/10.1163/ 1937240X-00002204

Beridze T, Edisherashvili T, Anderson C, Scheele F (2021) Ship sturgeon rediscovered in the Rioni River in Georgia. Oryx 55(1):9-9. https://doi.org/10.1017/S0030605320001003

Birstein VJ (2002) Sturgeon species and hybrids: can hybrids produce caviar. Environ Policy Law 32:210-2014

Boscari E, Barmintseva A, Pujolar JM, Doukakis P, Mugue N, Congiu L (2014) Species and hybrid identification of sturgeon caviar: a new molecular approach to detect illegal trade. Mol Ecol Res 14(3):489-498. https://doi.org/10.1111/1755-0998.12203

Boscari E, Vitulo N, Ludwig A, Caruso C, Mugue N, Suciu R, Onara FD (2017) Fast genetic identification of the Beluga sturgeon and its sought-after caviar to stem illegal trade. Food Control 75:145152. https://doi.org/10.1016/j.foodcont.2016.11.039

Clement M, Posada D, Crandall KA (2000) TCS: a computer program to estimate gene genealogies. Mol Ecol 9:1657-1659. https://doi. org/10.1046/j.1365-294x.2000.01020.x

Congiu L, Pujolar MJ, Forlani A, Cenadelli S, Dupanloup I, Barbisan F, Galli A, Fontana F (2011) Managing polyploidy in ex situ conservation genetics: the case of the critically endangered Adriatic sturgeon (Acipenser naccarii). PloS ONE 6(3):e18249. https://doi. org/10.1371/journal.pone.0018249

Excoffier L, Lischer HEL (2010) Arlequin Suite ver 3.5: a new series of programs to perform population genetics analyses under Linux and Windows. Mol Ecol Res 10:564-567. https://doi.org/10. 1111/j.1755-0998.2010.02847.x

Fjelldal PG, Wennevik V, Fleming IA, Hansen T, Glover KA (2014) Triploid (sterile) farmed Atlantic salmon males attempt to spawn with wild females. Aquacult Environ Interact 5:155-162. https:// doi.org/10.3354/aei00102

Gessner J, Williot P, Rochard E, Freyhof J, Kottelat M (2010) Acipenser sturio. IUCN Red List Threat Species. https://doi.org/10. 2305/IUCN.UK.2010-1

Guchmanidze A (2009) Current and historical status of sturgeon (Fam. Acipenseridae, Osteichthyes) in Georgia. In: Zazanashvuli $\mathrm{N}$, Mallon D (eds) Status and protection of globally threatened species in the Caucasus. CEPF, WWF. Contour Ltd, Tbilisi, pp $171-177$

Havelka M, Kašpar V, Hulák M, Flajšhans M (2011) Sturgeon genetics and cytogenetics: a review related to ploidy levels and interspecific hybridization. Folia Zool 60:93-103

Havelka M, Boscari E, Sergeev A, Mugue N, Congiu C, Arai K (2019) A new marker, isolated by dd RAD sequencing, detects Siberian and Russian sturgeon in hybrids. Animal Genet 50:115-116. https://doi.org/10.1111/age.12733

Hubbs CL (1955) Hybridization between fish species in nature. Syst Zool 4:1-20. https://doi.org/10.2307/2411933

IUCN (2010) Press Release, https://www.iucn.org/content/sturgeonmore-critically-endangered-any-other-group-species

Kumar S, Stecher G, Li M, Knyaz C, Tamura K (2018) MEGA X: molecular evolutionary genetics analysis across computing platforms. Mol Biol Evol 6(35):1547-1549. https://doi.org/10.1093/ molbev/msy096

Leigh JW, Bryant D (2015) Popart: Full-feature software for haplotype network construction. Methods Ecol Evol 6:1110-1116. https:// doi.org/10.1111/2041-210X.12410

Linhartová Z, Havelka M, Pšenička M, Flajšhans M (2018) Interspecific hybridization of sturgeon species affects differently their gonadal development. Czech J Anim Sci 63:1-10. https://doi.org/ 10.17221/37/2016-CJAS

Ludwig A, Lippold S, Debus L, Reinartz R (2009) First evidence of hybridization between endangered sterlets (Acipenser ruthenus) and exotic Siberian sturgeons (Acipenser baerii) in the Danube River. Biol Invasions 11:753-760. https://doi.org/10.1007/ s10530-008-9289-z

Mugue N, Barmintseva A, Schepetov D, Shalgimbayeva G, Isbekov K (2016) Complete mitochondrial genomes of the critically endangered Ship sturgeon Acipenser nudiventris from two seas. Mitochondrial DNA Part B 1:195-197. https://doi.org/10.1080/23802 359.2016.1144103

Variadilis L, Bolkvadze I, Khintibidze M, Zarkua Z, Gogotishvili M (1998) The Current State of the Georgian Black Sea Sturgeons. Kluwer Academic Publishers, Dordrecht, pp 235-241. https://doi. org/10.1007/978-94-011-5114-6_14

Publisher's Note Springer Nature remains neutral with regard to jurisdictional claims in published maps and institutional affiliations. 